\title{
Transurethral Resection of Prostate and the Role of Pharmacological Treatment with Dutasteride in Decreasing Surgical Blood Loss.
}

\author{
A.L. Pastore*, G. Palleschi*, V. Petrozza*, S. Mariani\#, M.A. Valentini\#, F. Barrese ${ }^{\#}$, M. \\ Cappa $^{\#}$, A.Carbone* \\ * Unit of Urology, Department of Medical and Surgical Sciences and Biotechnologies, \\ Faculty of Pharmacy and Medicine, Sapienza University of Rome, Latina (LT), Italy. \\ \# Unit of Urology, Fabia Mater Hospital, Rome, Italy
}

Introduction and aim of the study: Transurethral resection of prostate (TURP) still represents the gold standard in the surgical treatment of symptomatic benign prostatic hyperplasia (BPH). The most frequent complication is represented by intra- and perioperative bleeding. Preoperative use of 5 -alpha-reductase inhibitors (finasteride or dutasteride) to reduce surgical bleeding is still a topic of debate in literature. Previous studies provided favorable data on blood loss reduction by preoperative administration of finasteride or dutasteride. The aim of this study was to evaluate whether pretreatment with dutasteride for six weeks before surgery can reduce surgical blood loss.

Materials and methods: A total of 142 patients with BPH-who were to undergo TURPwere enrolled and randomized into two groups. The dutasteride group comprising of 71 patients, was treated with dutasteride $(0.5 \mathrm{mg} /$ day) for 6 weeks before surgery and the control group, comprising of other 71 patients, did not receive dutasteride. Blood loss was evaluated in terms of a reduction in the serum hemoglobin level $(\Delta \mathrm{Hb}$ and $\triangle H C T)$, and was estimated by measuring the $\mathrm{Hb}$ and hematocrit levels before and 24 hours after surgery.

Results: None of the patients treated with dutasteride reported any side effects. There was no statistically significant difference between the two groups with regard to age, prostate volume, resected prostate weight, PSA, and operation time. A significantly lower mean blood loss was observed in the dutasteride group compared to the control group $(\Delta \mathrm{Hb}=-1.29 \pm 0.81$ vs. $-1.83 \pm 1.25$, respectively, $\mathrm{p}<0.0027 ; \Delta \mathrm{HCT}=-5.67 \pm 2.58$ vs. $6.50 \pm 2.40$, respectively, $p<0.0491)$.

Conclusions: None of the previous studies had used dutasteride for 6 weeks before surgery. The results of the present study showed that treatment with dutasteride for 6 weeks before TURP reduces surgical bleeding. Although, in most cases the drop in $\mathrm{HCT} / \mathrm{Hgb}$ (while statistically significant) was not clinically significant, but it is important considering that we have enrolled a large number of patients (i.e., 71) in each group.

Table 1. Characteristics and Results of the Two Study Groups

\begin{tabular}{lccc}
\hline & $\begin{array}{c}\text { Dutasteride } \\
\text { group }(\mathrm{n}=71)\end{array}$ & $\begin{array}{c}\text { Control } \\
\text { group }(\mathrm{n}=71)\end{array}$ & $\begin{array}{c}\mathrm{p} \text {-Value } \\
\text { Student's } \\
\text { t-test }\end{array}$ \\
\hline Age (years) & $65.66 \pm 6.63 ; 66$ & $66.70 \pm 6.31 ; 67$ & 0.3400 \\
PSA (ng/mL) & $3.07 \pm 3.18 ; 2.2$ & $3.01 \pm 2.48 ; 2.15$ & 0.9004 \\
Prostate volume (mL) & $54.19 \pm 19.03 ; 49.5$ & $55.76 \pm 23.43 ; 50$ & 0.6619 \\
Resected prostate $(\mathrm{g})$ & $30.75 \pm 14.9 ; 27$ & $31.32 \pm 19.01 ; 26.5$ & 0.8427 \\
Hb before TURP (g/dL) & $14.27 \pm 1.06 ; 14.6$ & $14.27 \pm 1.01 ; 14.2$ & 0.0643 \\
Hb after TURP (g/dL) & $13.16 \pm 1.28 ; 13.5$ & $12.44 \pm 1.49 ; 12.7$ & $0.0022^{\mathrm{a}}$ \\
$\Delta$ Hb & $-1.29 \pm 0.81 ;-1.2$ & $-1.83 \pm 1.25 ;-1.8$ & $0.0027^{\mathrm{a}}$ \\
HCT before TURP & $43.57 \pm 3.01 ; 43.8$ & $33.1 \pm 2.94 ; 43.02$ & 0.2627 \\
HCT after TURP & $37.9 \pm 3.3 ; 39$ & $-6.50 \pm 2.2 ; 38.8$ & $0.0174^{\mathrm{a}}$ \\
$\Delta$ Hct & $-5.67 \pm 2.58 ;-4.1$ & $55.62 \pm 23.76 ; 50$ & $0.0491^{\mathrm{a}}$ \\
Operation time (minutes) & $58.4 \pm 19.5 ; 60$ & & 0.4473 \\
\hline
\end{tabular}

Values are represented as mean \pm SD (Standard Deviation); median.

$p<0.05$.

$\Delta \mathrm{Hb}=$ delta hemoglobin; $\Delta \mathrm{HCT}=$ delta hematocrit; $\mathrm{PSA}=$ prostate-specific antigen; TURP $=$ transurethral resection of the prostate. 